\title{
ON THE SOLUTION OF A NON-LINEAR PARABOLIC EQUATION WITH A FLOATING BOUNDARY ARISING IN A PROBLEM OF PLASTIC IMPACT OF A BEAM*
}

\author{
BY \\ THOMAS C. T. TING \\ Brown University, Providence, R. I.
}

\begin{abstract}
The deformation of a cantilever beam with strain rate sensitivity subjected to impact loading at its base has been studied in [11] by an approximate method in which the inertia forces in the plastic region are neglected. If these forces are taken into account, the equation of motion in the plastic region is a fourth order non-linear parabolic differential equation with a floating boundary, i.e. one whose position varies with time and must be found as part of the solution. A numerical solution of this equation is presented here. The results show that the bending moment in the plastic region varies nearly linearly. This result implies that the shear force is nearly constant in the plastic region, and hence that the inertia forces in the plastic region are small in comparison with the shear force in the same region.
\end{abstract}

1. Introduction. When the base of a cantilever beam is subjected to a severe impact, the subsequent permanent deformation may in some circumstances be analyzed by a treatment which neglects elastic vibrations and considers the plastic deformation to be governed by a strain rate-dependent yield stress. It has been shown [11] that such a treatment involves a deformation pattern with a plastic segment near the base of the cantilever beam and an outer segment which moves as a rigid body. The length $\zeta(t)$ of the plastic segment varies as time changes, and hence gives a "floating" boundary (see Fig. 1). The non-dimensional coordinate $x$ is so chosen that $x=0$ at base and $x=1$ at tip. For convenience, the non-dimensional velocity $v$ and time $t$ are introduced. They are related to the actual velocity $V$ and time $T$ by following relations:

$$
\begin{aligned}
& v=\left(m l / M_{0}\right)^{1 / 2} V \\
& t=\left(M_{0} / m l^{3}\right)^{1 / 2} T
\end{aligned}
$$

where $m$ is the mass per unit length of the beam, $l$ the length of the beam and $M_{0}$ the static yield moment of the beam. By writing the equation of motion for the plastic region, it is shown in the next section that the velocity $v(x, t)$ in this region must satisfy the equation

$$
\left[\left(v_{x x} / \alpha\right)^{1 / p}\right]_{x x}+v_{t}=0
$$

where a subscript $x$ stands for $\partial / \partial x$ and a subscript $t$ for $\partial / \partial t$. $p$ and $\alpha$ are constants in the expression of strain rate dependence of the yield stress, Eq. (10). The initial conditions are

$$
v(x, 0)=v_{0}=\text { const., } \zeta(0)=0
$$

and the boundary conditions are

$$
v(0, t)=v_{0}[1-f(t)], \quad v_{x}(0, t)=v_{x x}(\zeta, t)=0
$$

*Received November 23, 1962. The results presented in this paper were obtained in the course of research sponsored by the Office of Naval Research Contract Nonr 562(10) with Brown University. 


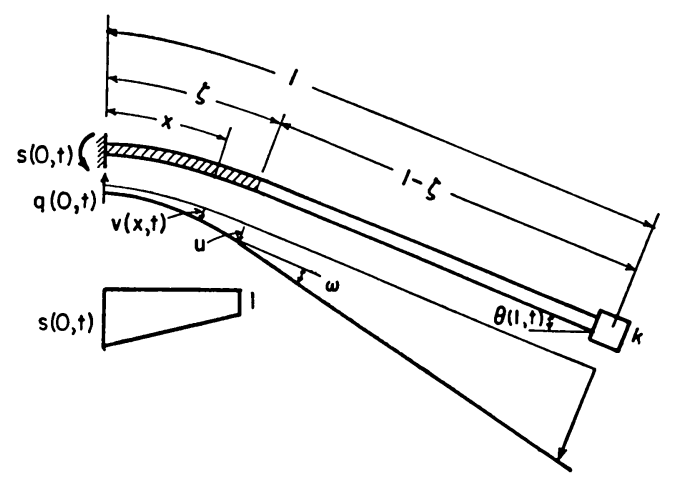

(a)

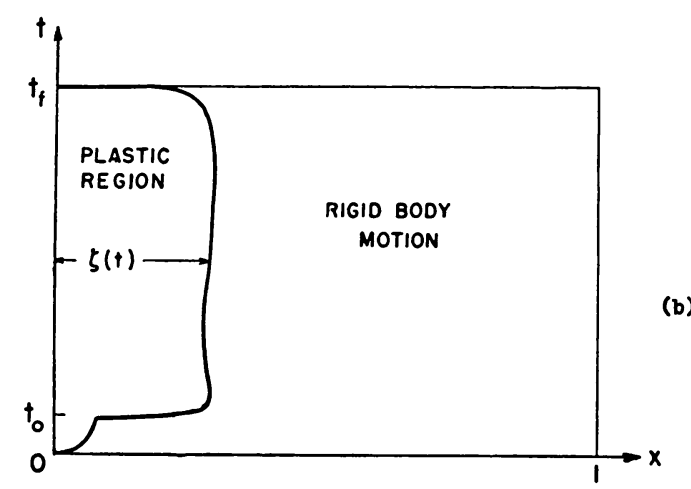

FIg. 1 Typical State of Deformation of the Beam

where $f(t)$ is a continuous function of $t$ with the condition that

and

$$
f(t)=0 \text { for } t \leq 0
$$

$$
f(t)=1 \text { for } t \geq t_{0}=\text { positive constant. }
$$

Two more conditions for the floating boundary are provided by the equations of conservation of angular and linear momentum of the whole beam. They are:

$$
\begin{gathered}
\left(\frac{1}{2}+k\right) v_{0} f(t)-\int_{0}^{t} s(0, \tau) d \tau=\Phi_{1}(t) \\
(1+k) v_{0} f(t)-\int_{0}^{t} q(0, \tau) d \tau=\Phi_{2}(t) \cos \theta(1, t)
\end{gathered}
$$

where $k$ is the mass ratio (the ratio of the tip mass to the mass of the beam) and

$$
\begin{gathered}
\Phi_{1}(t)=\int_{0}^{\zeta}(1-x)\left[k+\frac{1}{6}(1-x)(2+x)\right] v_{x x}(x, t) d x \\
\Phi_{2}(t)=\int_{0}^{\zeta}(1-x)\left[k+\frac{1}{2}(1-x)\right] v_{x x}(x, t) d x \\
\theta(1, t)=\int_{0}^{t} v_{x}(\zeta, \tau) d \tau
\end{gathered}
$$




$$
\begin{gathered}
s(x, t)=1+\left(v_{x x} / \alpha\right)^{1 / p} \\
q(x, t)=-s_{x}(x, t)=-\left[\left(v_{x x} / \alpha\right)^{1 / p}\right]_{x} .
\end{gathered}
$$

The notations $s$ and $q$ are the non-dimensional bending moment and shear force of a cross section respectively. They are related to the actual bending moment $M$ and the shear force $Q$ by $s=M / M_{0}$ and $q=Q l / M_{0}$. The detailed derivations of the above equations are shown in the next section.

The solution of the non-linear fourth order parabolic equation, Eq. (1) presents considerable difficulties. An analytic solution seems out of the question. Very few numerical solutions of non-linear higher order parabolic equations have been presented. and no solution of a problem involving also a floating boundary appears to have been given. Ehrlich [1], and Douglas and Gallie [2] have discussed the linear heat equation with a moving boundary. Young and Ehrlich [3] have considered a non-linear second order parabolic equation with fixed boundaries. They employed various finite difference methods. Conte and Royster [4] have studied a finite difference approximation for the solution of the fourth order parabolic equation $v_{x x x x}+v_{t}=0$. It is linear and the boundaries are fixed.

There are several finite difference methods for solving the linear or quasi-linear parabolic differential equations. The forward difference method provides an explicit expression for each step but the mesh ratio has to be restricted to assure the stability of the numerical solution (see for example [5] and [6]). Especially in the case of higher order parabolic equations, the mesh ratio must be so small that the time increment has to be taken inconveniently small. The Crank-Nicolson method and the backward difference method are stable for all mesh ratios, but the unknown quantities appear in implicit form (see [7], [8] and [9]). This requires solving a system of simultaneous equations which are linear or not depending on the original parabolic equation. DuFort and Frankel [10] obtained a method which gives an explicit expression for the unknown quantities while the stability is still assured for all mesh ratio. Nevertheless, the ratio is still restricted if the convergence of the solution is to be assured. In any case, to use a finite difference method to solve Eq. (1) presents many difficulties. Besides those mentioned, since a finite difference expression for (1) requires at least five points in the $x$-direction, there is a further difficulty in the region near $t=0$ (see Fig. 1).

The numerical technique presented here is similar to the backward difference method in nature but uses the integral representation instead of the difference equation. The technique proves to be very effective for this particular problem. The results are compared with those obtained from the simplified equations of motion in which the inertia forces in the plastic region are neglected (see [11]). The closeness of values of $v_{x x}(0, t)$, $s(0, t), q(0, t)$ and $\theta(1, t)$ obtained from these two solutions is remarkable for the several examples calculated. This result shows that the neglect of the inertia forces in the plastic region is permissible as far as $v_{x x}(0, t), s(0, t), q(0, t)$ and $\theta(1, t)$ are concerned.

2. Equations of motion. A cantilever beam of uniform cross section with an attached tip mass and with an initial velocity of $v_{0}$ has its base brought to rest by a collision with an anvil [11]. The velocity of the base is specified by $v(0, t)=v_{0}[1-f(t)]$, where $f(t)$ is a continuous function of $t$ subject to the conditions (4). For $0 \leq t \leq t_{0}, f(t)$ can be of any form (see Fig. 2).

The beam is assumed of rigid-plastic material with strain rate sensitivity. The strain rate property is taken to be expressed by the moment-curvature relation (see [11]) 


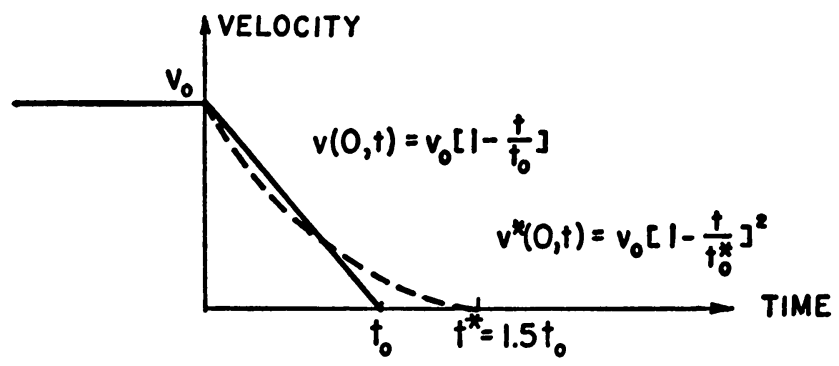

Fig. 2. Various Forms of Velocity at the Base.

$$
v_{x x}=\kappa_{t}=\alpha(s-1)^{p}
$$

or

$$
s=1+\left(v_{x x} / \alpha\right)^{1 / p}
$$

where $\kappa$ is the non-dimensional curvature, which is equivalent to the actual curvature multiplied by $l$. This is Eq. (8): For convenience, we will put

$$
s=1+A
$$

where

$$
A=\left(v_{x x} / \alpha\right)^{1 / p}=\left(\kappa_{t} / \alpha\right)^{1 / p}
$$

or

$$
\kappa_{t}(x, t)=v_{x x}(x, t)=\alpha[A(x, t)]^{p} .
$$

As stated in [11], if the change of the velocity at the base is not too large, the deformation configuration consists of a plastic segment adjacent to the base and the remaining segment of the beam which is in a rigid body motion (see Fig. 1). The moment $s(x, t)$ in the plastic region exceeds the static yield moment everywhere, i.e. $s(x, t)>1$, and the rate of curvature $\kappa_{t}(x, t)$ obeys (10c). The equation of motion for a beam of uniform cross section is

$$
s_{x x}+v_{t}=0 .
$$

Inserting (10) in (11), one gets (1), which is the equation of motion in the plastic region.

The initial conditions (2) and the boundary conditions (3) are physically obvious. At the floating boundary $x=\zeta, s(\zeta, t)=1$; hence $v_{x x}(\zeta, t)=0$ by $(8) . \zeta(0)$ must be zero; otherwise, (1) could not be satisfied at $t=0$ since $v_{t}(x, 0) \neq 0$ while $v(x, 0)=v_{0}=$ constant.

The equations of angular and linear momentum of the whole beam are (see Fig. 1), respectively,

$$
\begin{gathered}
\int_{0}^{t} s(0, \tau) d \tau \cong v_{0}\left(\frac{1}{2}+k\right)-\int_{0}^{1} x v(x, t) d x-k v(1, t) \\
=v_{0}\left(\frac{1}{2}+k\right)-u\left(\frac{1-\zeta^{2}}{2}+k\right)-\omega(1-\zeta)\left[\frac{1-\zeta}{2}\left(1-\frac{1-\zeta}{3}\right)+k\right] \\
-\int_{0}^{\zeta} x v d x
\end{gathered}
$$




$$
\begin{aligned}
& \int_{0}^{t} q(0, \tau) d \tau \cong v_{0}(1+k)-\left[\int_{0}^{1} v(x, t) d x+k v(1, t)\right] \cos \theta(1, t) \\
& =v_{0}(1+k)-\left[u(1-\zeta+k)+\omega(1-\zeta)\left(\frac{1-\zeta}{2}+k\right)+\int_{0}^{\zeta} v d x\right] \cos \theta(1, t)
\end{aligned}
$$

where $u=v(\zeta, t), \omega=v_{x}(\zeta, t), q(x, t)=-s_{x}(x, t)=$ the shear force, and $\theta(x, t)$ is the slope angle of the beam. The correction for the geometric change of the beam by the use of $\cos \theta(1, t)$ makes use of the approximations:

$$
\begin{array}{ll}
0 \leq x \leq \zeta: & \theta(x, t) \cong 0 \\
\zeta \leq x \leq 1: & \theta(x, t) \cong \theta(1, t) .
\end{array}
$$

If we use the relation

$$
\begin{aligned}
u & =v_{0}[1-f(t)]+\int_{0}^{\zeta} v_{x} d x \\
& =v_{0}[1-f(t)]+\zeta \omega-\int_{0}^{\zeta} x v_{x x} d x,
\end{aligned}
$$

equations (12), after integrating by parts, can be reduced to

$$
\begin{aligned}
& \int_{0}^{t} s(0, \tau) d \tau= v_{0}\left(\frac{1}{2}+k\right) f(t)-\int_{0}^{\zeta}(1-x)\left[k+\frac{1}{6}(1-x)(2+x)\right] v_{x x}(x, t) d x \\
& \int_{0}^{t} q(0, \tau) d \tau=v_{0}(1+k)\{1-[1-f(t)] \cos \theta(1, t)\} \\
&-\left\{\int_{0}^{\zeta}(1-x)\left[k+\frac{1}{2}(1-x)\right] v_{x x}(x, t) d x\right\} \cos \theta(1, t) .
\end{aligned}
$$

This is Eq. (5) with the term $\{1-[1-f(t)] \cos \theta(1, t)\}$ replaced by $f(t)$, since $f(t)=1$ for $t \geq t_{0}$ and $\cos \theta(1, t) \cong 1$ for $0 \leq t \leq t_{0}$. have

In the rigid body portion, $v_{x}(1, t)=v_{x}(\zeta, t)$. Since $\theta_{t}(1, t)=v_{x}(1, t)=v_{x}(\zeta, t)$, we

$$
\theta(1, t)=\int_{0}^{t} v_{x}(\zeta, \tau) d \tau
$$

This is Eq. (7).

Thus, Eqs. (1) through (9) give a complete description of the impact of a cantilever beam with strain rate sensitivity. Following is presented a numerical technique for the solution of this system of equations.

3. Numerical solution. Equation (1) can be integrated four times with respect to $x$ if $v_{t}(x, t)$ is known for a fixed $t$. Four initial values are necessary. If we know $q(0, t)$ and $A(0, t)$ for this fixed time $t$, and use the first two conditions of (3), Eq. (1) in integral form is

$v(x, t)=v(0, t)+\int_{0}^{x} \int_{0}^{\sigma} \alpha\left\{A(0, t)-\int_{0}^{\lambda}\left[q(0, t)+\int_{0}^{\eta} v_{t}(\xi, t) d \xi\right] d \eta\right\}^{\nu} d \lambda d \sigma$. 
In addition, we approximate $v_{t}(x, t)$ by

$$
v_{t}(x, t)=[v(x, t)-v(x, t-\Delta t)] / \Delta t
$$

where $\Delta t$ is the time increment for the numerical calculation.

Suppose we know the value of $v(x, t-\Delta t)$ for all $x$ at $t-\Delta t$ and try to find $v(x, t)$ for all $x$ at $t$. The numerical procedure consists of two main stages as follows. First stage: Assume an approximate value for $q(0, t)$ and $\kappa_{t}(0, t)$ and solve equation $(13) . A(0, t)$ is related to $\kappa_{t}(0, t)$ by $(10 \mathrm{~b})$. Second stage: Correct the approximate values $q(0, t), \kappa_{t}(0, t)$ by (5). The detailed procedure are explained separately in the following.

First stage. Since $q(0, t)$ and $A(0, t)$ are assumed to be known, Eq. (13a) and (13b) gives an integral equation for $v(x, t)$ for a fixed $t$. The solution of (13) can be obtained by a method of successive approximation. Suppose that we replace $v_{t}(x, t)$ in (13a) by an initial approximation $v_{t}^{(0)}(x, t)$. Then (13a) determines an approximation $v^{(1)}(x, t)$ and $(13 b)$ gives

$$
v_{t}^{(1)}(x, t)=\left[v^{(1)}(x, t)-v(x, t-\Delta t)\right] / \Delta t
$$

By substituting this approximation into (13a), we then obtain the next approximation $v^{(2)}(x, t)$ and hence $v_{t}^{(2)}(x, t)$ by $(13 \mathrm{~b})$. We continue this process until the desired accuracy for $v_{t}(x, t)$ is obtained. We will show under what conditions the successive approximations actually converge toward the solution of (13).

In order to see it more clearly, we write (13a) for each integration separately. Moreover, we do not restrict the lower limit of the integrations to be zero. Instead, we take $x=x_{i}$ as our lower limit for generality. Thus, for the $(n-1)$ th approximate value of $v_{t}(x, t)$, we have $(n)$ th approximate values of each integral expressed as follows:

$$
\begin{gathered}
q^{(n)}(x, t)=q\left(x_{i}, t\right)+\int_{x_{i}}^{x} v_{t}^{(n-1)}(\xi, t) d \xi \\
A^{(n)}(x, t)=A\left(x_{i}, t\right)-\int_{x_{i}}^{x} q^{(n)}(\xi, t) d \xi \\
v_{x x}^{(n)}(x, t)=\kappa_{t}^{(n)}(x, t)=\alpha\left[A^{(n)}(x, t)\right]^{p} \\
v_{x}^{(n)}(x, t)=\theta_{t}^{(n)}(x, t)=\theta_{t}\left(x_{i}, t\right)+\int_{x_{i}}^{x} \kappa_{t}^{(n)}(\xi, t) d \xi \\
v^{(n)}(x, t)=v\left(x_{i}, t\right)+\int_{x_{i}}^{x} \theta_{t}^{(n)}(\xi, t) d \xi .
\end{gathered}
$$

Equation (13b) then gives all

$$
v_{t}^{(n)}(x, t)=\left[v^{(n)}(x, t)-v(x, t-\Delta t)\right] / \Delta t .
$$

When $x_{i}=0$, the system (14) reduces to an iteration scheme for (13).

Now, let $\delta v_{t}^{(n-1)}(x, t)$ be the error of the $(n-1)$ th approximation $v_{t}^{(n-1)}(x, t)$ to the true value $v_{t}(x, t)$, i.e.,

$$
v_{t}^{(n-1)}(x, t)=v_{t}(x, t)-\delta v_{t}^{(n-1)}(x, t) .
$$

Substituting (15) into (14a), one obtains 


$$
q^{(n)}(x, t)=q(x, t)-\int_{x_{i}}^{x} \delta v_{t}^{(n-1)}(\xi, t) d \xi
$$

(14b) and (14c) become

$$
\begin{gathered}
A^{(n)}(x, t)=A(x, t)+\int_{x_{i}}^{x} \int_{x_{i}}^{\eta} \delta v_{t}^{(n-1)}(\xi, t) d \xi d \eta, \\
\kappa_{t}^{(n)}(x, t) \cong \kappa_{t}(x, t)+\alpha p[A(x, t)]^{\nu-1} \int_{x_{i}}^{x} \int_{x_{i}}^{\eta} \delta v_{t}^{(n-1)}(\xi, t) d \xi d \eta,
\end{gathered}
$$

if the higher order terms are neglected. Similar expressions can be obtained for (14d) and $(14 \mathrm{e})$. For Eq. (14f) one obtains

$$
v_{t}^{(n)}(x, t) \cong v_{t}(x, t)+\frac{\alpha p}{\Delta t} \int_{x_{i}}^{x} \int_{x_{i}}^{\sigma}[A(\lambda, t)]^{p-1} \int_{x_{i}}^{\lambda} \int_{x_{i}}^{\eta} \delta v_{t}^{(n-1)}(\xi, t) d \xi d \eta d \lambda d \sigma .
$$

Hence, by the definition of (15), we have

$$
\delta v_{t}^{(n)}(x, t) \cong-\frac{\alpha p}{\Delta t} \int_{x_{i}}^{x} \int_{x_{i}}^{\sigma}[A(\lambda, t)]^{p-1} \int_{x_{i}}^{\lambda} \int_{x_{j}}^{\eta} \delta v_{t}^{(n-1)}(\xi, t) d \xi d \eta d \lambda d \sigma .
$$

The successive approximation will converge if

$$
\left|\delta v_{t}^{(n)}(x, t)\right|<\left|\delta v_{t}^{(n-1)}(x, t)\right|
$$

It remains to determine the conditions under which (16) is satisfied.

In our impact problem, $A(x, t)$ is a positive, monotonic decreasing function of $x$ (see Fig. 7). Hence, (16) is satisfied if

$$
\frac{\alpha p}{\Delta t}\left[A\left(x_{i}, t\right)\right]^{p-1} \int_{x_{i}}^{x} \int_{x_{i}}^{\sigma} \int_{x_{j}}^{\lambda} \int_{x_{i}}^{\eta}\left|\delta v_{t}^{(n-1)}(\xi, t)\right| d \xi d \eta d \lambda d \sigma<\left|\delta v_{t}^{(n-1)}(x, t)\right| .
$$

Consider an error function of the form

$$
\left|\delta v_{t}^{(n-1)}(x, t)\right|=\left|\epsilon_{1}\left(x-x_{i}\right)\right|
$$

where $\epsilon_{1}$ is a constant. Then, (17) gives

$$
\frac{p \alpha\left(x-x_{i}\right)^{4}}{5 ! \Delta t}\left[A\left(x_{i}, t\right)\right]^{p-1}<1 .
$$

Similarly, if $\left|\delta v_{t}^{(n-1)}(x, t)\right|=\left|\epsilon_{\nu}\left(x-x_{i}\right)^{\nu}\right|$ where $\nu$ is an integer and $\epsilon_{\nu}$ are constants, (17) gives

$$
\frac{p \alpha\left(x-x_{i}\right)^{4}}{(\nu+1)(\nu+2)(\nu+3)(\nu+4) \Delta t}\left[A\left(x_{i}, t\right)\right]^{p-1}<1 \text { for } \nu>1 .
$$

It is seen that (19) implies (20). If $\left(x-x_{i}\right)$ is sufficiently small, then the error function in Taylor's expansion $\delta v_{t}^{(n-1)}(x, t)=\sum_{v=1}^{\infty} \epsilon_{\nu}\left(x-x_{i}\right)^{\prime}$ is dominated by its first term, i.e. (18). Hence (19) provides the condition for the convergence of successive approximation if $\left(x-x_{i}\right)$ is sufficiently small.

In the numerical integration of (13a), an equal interval of $\Delta x$ is taken in the $x$-direction. The $j$-th point $x_{i}$ from $x=0$ is $x_{i}=j \Delta x(j=0,1,2, \cdots)$. Equation (14) enables one to calculate $v_{t}(x, t)$ point by point for $x=x_{1}, x_{2}, x_{3}, \cdots$. Thus, after having found $v_{t}\left(x_{i}, t\right)$, one proceeds to find $v_{t}\left(x_{i+1}, t\right)$ by putting $x=x_{i+1}=x_{i}+\Delta x$ in (14). The 
convergence of the successive approximation is assured if

$$
\frac{p \alpha(\Delta x)^{4}}{5 ! \Delta t}\left[A\left(x_{i}, t\right)\right]^{p-1}<1
$$

Which is obtained by replacing $\left(x-x_{i}\right)$ by $\Delta x$ in (19).

The calculation starts from $x=x_{1}=\Delta x$. We know $v_{t}(0, t)$. To calculate $v_{t}\left(x_{1}, t\right)$ by (14), it is natural to take $v_{t}^{(0)}\left(x_{1}, t\right)=v_{t}(0, t)$ as an initial approximation. In a similar way, to calculate $v_{t}\left(x_{2}, t\right), v_{t}^{(0)}\left(x_{2}, t\right)=v_{t}\left(x_{1}, t\right)$ is taken as an initial approximation. The process is repeated until for a certain $j$, say $\lambda, A\left(x_{\lambda}, t\right)>0$ while $A\left(x_{\lambda+1}, t\right)<0$. In other words, $A(\zeta, t)=0$ lies between $x_{\lambda}$ and $x_{\lambda+1}$ and this implies that the floating boundary $\zeta$ lies between these two points. Assuming a linear variation of $A(x, t)$ between $x_{\lambda}$ and $x_{\lambda+1}$, one obtains the value of $\zeta$ by

$$
\zeta=x_{\lambda}+\frac{A\left(x_{\lambda}, t\right)}{A\left(x_{\lambda}, t\right)-A\left(x_{\lambda+1}, t\right)} \Delta x .
$$

In evaluating the integrals in (14), the ordinary trapezoidal method is used for (14a) and (14b). By assuming a linear variation between $A\left(x_{i}, t\right)$ and $A\left(x_{i+1}, t\right)$, one obtains explicit expressions for the integrals of (14d) and (14e) as follows:

$$
\theta_{t}\left(x_{i+1}, t\right)=\theta_{t}\left(x_{i}, t\right)+\frac{\kappa_{t}\left(x_{i}, t\right)}{p+1}\left(\frac{1-r^{p+1}}{1-r}\right) \Delta x
$$

$v\left(x_{i+1}, t\right)=v\left(x_{i}, t\right)+\theta_{t}\left(x_{i}, t\right) \Delta x+\frac{\kappa_{t}\left(x_{i}, t\right)}{(p+1)(1-r)}\left[1-\frac{1-r^{p+2}}{(p+2)(1-r)}\right](\Delta x)^{2}$

where $r=A\left(x_{i+1}, t\right) / A\left(x_{i}, t\right)$. For simplicity, we have omitted the superscript $(n)$.

At the floating boundary $x=\zeta, A(\zeta, t)=\kappa_{t}(\zeta, t)=0$. By putting $r=0$ and replacing $x_{i+1}$ by $\zeta$ and $\Delta x$ by $\left(\zeta-x_{i}\right)$ in (22), we obtain the values of $\theta_{t}(\zeta, t)$ and $v(\zeta, t)$. Equation (14f) then gives $v_{t}(\zeta, t)$ and (14a) gives $q(\zeta, t)$. All the calculations are completed for this stage, and we can go to the next stage to correct the assumed values $q(0, t)$ and $\kappa_{t}(0, t)$.

Second stage. With all the values obtained in the first stage, we can put them into equations (5a) and $(5 \mathrm{~b})$ to check the equalities. These two equations are not satisfied if the assumed values $q(0, t)$ and $\kappa_{t}(0, t)$ are not correct. In this second stage, we will derive expressions for the correction of $q(0, t)$ and $\kappa_{t}(0, t)$.

Suppose that the equalities in $(5 \mathrm{a})$ and $(5 \mathrm{~b})$ are not identically satisfied so that

$$
\begin{gathered}
\left(\frac{1}{2}+k\right) v_{0} f(t)-\int_{0}^{t} s(0, \tau) d \tau-\Phi_{1}(t)=\Delta I_{1} \\
(1+k) v_{0} f(t)-\int_{0}^{t} q(0, \tau) d \tau-\Phi_{2}(t) \cos \theta(1, t)=\Delta I_{2}
\end{gathered}
$$

where $\Delta I_{1}$ and $\Delta I_{2}$ are not both zero. We shall find what changes should be made of $q(0, t)$ and $\kappa_{t}(0, t)$ to bring both $\Delta I_{1}$ and $\Delta I_{2}$ to zero or to sufficiently small values.

Let $q(0, t)$ and $\kappa_{t}(0, t)$ have small variations $\delta q$ and $\delta \kappa_{t}$, respectively. The magnitudes of $\Phi_{1}(t)$ and $\Phi_{2}(t)$ in (23) will have small variations $\delta \Phi_{1}$ and $\delta \Phi_{2}$, according to $\delta q$ and $\delta \kappa_{t}$. The values of integrals in (23) will also vary according to $\delta q$ and $\delta \kappa_{t}$. We have to find their relations before we can determine $\delta q$ and $\delta \kappa_{t}$ in terms of $\Delta I_{1}$ and $\Delta I_{2}$. 
Since $\kappa_{t}=\alpha A^{p}$, the relation between $\delta \kappa_{t}$ and $\delta A$ is

$$
\delta A=\frac{A(0, t)}{p \kappa_{t}(0, t)} \delta \kappa_{t} .
$$

The integral in $(23 \mathrm{~b})$ is

$$
\int_{0}^{t} q(0, \tau) d \tau=\int_{0}^{t-\Delta t} q(0, \tau) d \tau+\left[\frac{q(0, t-\Delta t)+q(0, t)}{2}\right] \Delta t .
$$

Hence, the variation of this integral due to $\delta q$ of $q(0, t)$ is $\delta q / 2 \Delta t$. Similarly, the variation of the integral in (23a) is $\delta A / 2 \Delta t$, or $A(0, t) \delta \kappa_{t} \Delta t / 2 p \kappa_{t}(0, t)$ by (24). Now, the right hand sides of Eqs. (23) can be reduced to zero if

$$
\begin{gathered}
\frac{\delta q}{2} \Delta t+\delta \Phi_{1}=\Delta I_{1} \\
\frac{A(0, t)}{2 p \kappa_{t}(0, t)} \delta \kappa_{\iota} \Delta t+\delta \Phi_{2} \cos \theta(1, t)=\Delta I_{2}
\end{gathered}
$$

where, as stated before, $\delta \Phi_{1}, \delta \Phi_{2}$ are variations of $\Phi_{1}, \Phi_{2}$ due to $\delta q$ and $\delta \kappa_{t}$ respectively. Here, we have neglected the variation of $\cos \theta(1, t)$. It remains to find $\delta \Phi_{1}, \delta \Phi_{2}$ in terms of $\delta q$ and $\delta \kappa_{t}$.

The equation of equilibrium for the plastic segment of the beam as a whole is

$$
A(0, t)=q(0, t) \zeta+\int_{0}^{\zeta}(\zeta-x) v_{t}(x, t) d x
$$

The last term of Eq. (26) was neglected in the analysis in [11] so that $A(x, t)$ is linear in $x$ in the plastic region. In our analysis, $A(x, t)$ is no longer linear in $x$. However, it is very close to it, as shown in several examples calculated (see, for instance, Fig. 7). This indicates that the integral in (26) is of negligible magnitude compared with the term $q(0, t) \zeta$. If the variation of this integral is neglected, we have

$$
\delta A \cong \delta[q(0, t) \zeta]=q(0, t) \delta \zeta+\zeta \delta q
$$

or, by (24),

$$
\delta \zeta=\frac{1}{q(0, t)}\left[\frac{A(0, t)}{p \kappa_{\iota}(0, t)} \delta \kappa_{t}-\zeta \delta q\right] .
$$

Now, due to the near linearity of $A(x, t)$ as stated above, we write

$$
A(x, t)=A(0, t)\left(1-\frac{x}{\zeta}\right)+\Delta A(x, t)
$$

where $\Delta A(x, t) \ll A(x, t)$. Hence

$$
v_{x x}(x, t)=\kappa_{t}(x, t)=\alpha[A(x, t)]^{p}=\kappa_{t}(0, t)\left(1-\frac{x}{\zeta}\right)^{p}+\Delta v_{x x}(x, t),
$$

where $\Delta v_{x x}(x, t) \ll v_{x x}(x, t)$. Inserting (28) in (5), we have

$$
\begin{aligned}
& \Phi_{1}(t)=\kappa_{t}(0, t) F_{1}(\zeta)+\Delta \Phi_{1} \\
& \Phi_{2}(t)=\kappa_{t}(0, t) F_{2}(\zeta)+\Delta \Phi_{2}
\end{aligned}
$$


where

$$
\begin{aligned}
F_{1}(\zeta) & =\int_{0}^{\zeta}(1-x)\left[k+\frac{1}{6}(1-x)(2+x)\right]\left(1-\frac{x}{\zeta}\right)^{p} d x \\
& =\left(\frac{1}{3}+k\right) \frac{\zeta}{p+1}-\frac{(1+2 k) \zeta^{2}}{2(p+1)(p+2)}+\frac{\zeta^{4}}{(p+1)(p+2)(p+3)(p+4)} \\
F_{2}(\zeta) & =\int_{0}^{\zeta}(1-x)\left[k+\frac{1}{2}(1-x)\right]\left(1-\frac{x}{\zeta}\right)^{p} d x \\
& =\left(\frac{1}{2}+k\right) \frac{\zeta}{p+1}-\frac{(1+k) \zeta^{2}}{(p+1)(p+2)}+\frac{\zeta^{3}}{(p+1)(p+2)(p+3)}
\end{aligned}
$$

and $\Delta \Phi_{1}, \Delta \Phi_{2}$ are of small magnitude compared with $\Phi_{1}(t)$ and $\Phi_{2}(t)$ respectively. If the variations of the last terms of (29) are neglected, we have, with the use of (27),

$$
\begin{aligned}
& \delta \Phi_{1}=F_{1}(\zeta) \delta \kappa_{t}+\frac{\kappa_{t}(0, t)}{q(0, t)} \frac{d F_{1}}{d \zeta}\left[\frac{A(0, t)}{p \kappa_{t}(0, t)} \delta \kappa_{t}-\zeta \delta q\right] \\
& \delta \Phi_{2}=F_{2}(\zeta) \delta \kappa_{t}+\frac{\kappa_{t}(0, t)}{q(0, t)} \frac{d F_{2}}{d \zeta}\left[\frac{A(0, t)}{p \kappa_{t}(0, t)} \delta \kappa_{t}-\zeta \delta q\right]
\end{aligned}
$$

After substituting (30) into (25), we obtain $\delta q$ and $\delta \kappa_{t}$ in terms of $\Delta I_{1}$ and $\Delta I_{2}$.

Thus, for given $\Delta I_{1}, \Delta I_{2}$, we calculate $\delta q$ and $\delta \kappa_{t}$. Take $q(0, t)+\delta q$ and $\kappa_{t}(0, t)+\delta \kappa_{t}$ as new approximate values and the first stage is repeated. This process is continued until $\Delta I_{1}$ and $\Delta I_{2}$ are both zero, or $\delta q$ and $\delta \kappa_{t}$ are very small compared with $q(0, t)$ and $\kappa_{t}(0, t)$ respectively.

4. Some details of the numerical calculations. At $t=0, \zeta=0$ and $v(x, 0)=$ const. by (2). Hence $v_{x}(0,0)=v_{x x}(0,0)=\kappa_{t}(0,0)=A(0,0)=0 . \theta(1,0)=\theta_{t}(1,0)=0$ by $(7)$ and $s(0,0)=1$ by $(8)$. The initial shear $q(0,0)$ can be obtained by differentiating (5) and taking the limit $t \rightarrow 0$. Note that

$$
\begin{aligned}
\frac{d \Phi_{1}}{d t}=\int_{0}^{\zeta}(1-x)\left[k+\frac{1}{6}(1-x)(2\right. & +x)] v_{x x t}(x, t) d x \\
& +(1-\zeta)\left[k+\frac{1}{6}(1-\zeta)(2+\zeta)\right] v_{x x}(\zeta, t) \zeta_{t}(t) .
\end{aligned}
$$

Since $v_{x x}(\zeta, t)=0$ by (3), and $\zeta \rightarrow 0$ as $t \rightarrow 0$, we have

$$
\begin{aligned}
\lim _{t \rightarrow 0} \frac{d \Phi_{1}}{d t} & =\lim _{\zeta \rightarrow 0} \int_{0}^{\zeta}(1-x)\left[k+\frac{1}{6}(1-x)(2+x)\right] v_{x x t}(x, t) d x \\
& =\left(k+\frac{1}{3}\right) \lim _{\zeta \rightarrow 0} \int_{0}^{\zeta} v_{x x t}(x, t) d x .
\end{aligned}
$$

Therefore, differentiation of (5a) with $t \rightarrow 0$ gives

$$
\left(\frac{1}{2}+k\right) v_{0} f_{t}(0)-1=\left(k+\frac{1}{3}\right) \lim _{\zeta \rightarrow 0} \int_{0}^{\zeta} v_{x x t}(x, t) d x
$$

Similarly, differentiation of $(5 \mathrm{~b})$ with $t \rightarrow 0$ gives

$$
(1+k) v_{0} f_{t}(0)-q(0,0)=\left(k+\frac{1}{2}\right) \lim _{\zeta \rightarrow 0} \int_{0}^{\zeta} v_{x x t}(x, t) d x
$$


By eliminating the integral term in (31), we get

$$
q(0,0)=\frac{v_{0} f_{t}(0)(k+1 / 4)+(k+1 / 2)}{3 k+1}
$$

At each step, to start the first stage of calculation, an approximate value of $q(0, t)$ and $\kappa_{t}(0, t)$ has to be chosen. This can be done by taking

$$
q(0, t) \cong q(0, t-\Delta t)+[q(0, t-\Delta t)-q(0, t-2 \Delta t)]
$$

or

$$
q(0, t) \cong 2 q(0, t-\Delta t)-q(0, t-2 \Delta t)
$$

and

$$
\kappa_{t}(0, t) \cong 2 \kappa_{t}(0, t-\Delta t)-\kappa_{t}(0, t-2 \Delta t)
$$

as long as the time increment $\Delta t$ is not altered. Special methods have to be devised to determine these approximate values at $t=\Delta t$ and also at steps where $\Delta t$ differs from their preceding steps. These are presented below.

Asymptotic solutions of $q(0, t)$ and $\kappa_{t}(0, t)$ as $t \rightarrow 0$, based on the simplified equations of motion obtained by neglecting the inertia forces in the plastic region, have been shown in [11]. They are

$$
\begin{array}{ll}
q(0, t) \sim q_{0}+\frac{3(2 k+1)}{2(3 k+1)}\left[\frac{c q_{0} t}{\alpha}\right]^{1 /(p+1)} & \text { as } t \rightarrow 0 \\
\kappa_{t}(0, t) \sim \alpha\left[\frac{c q_{0} t}{\alpha}\right]^{p /(p+1)} & \text { as } t \rightarrow 0
\end{array}
$$

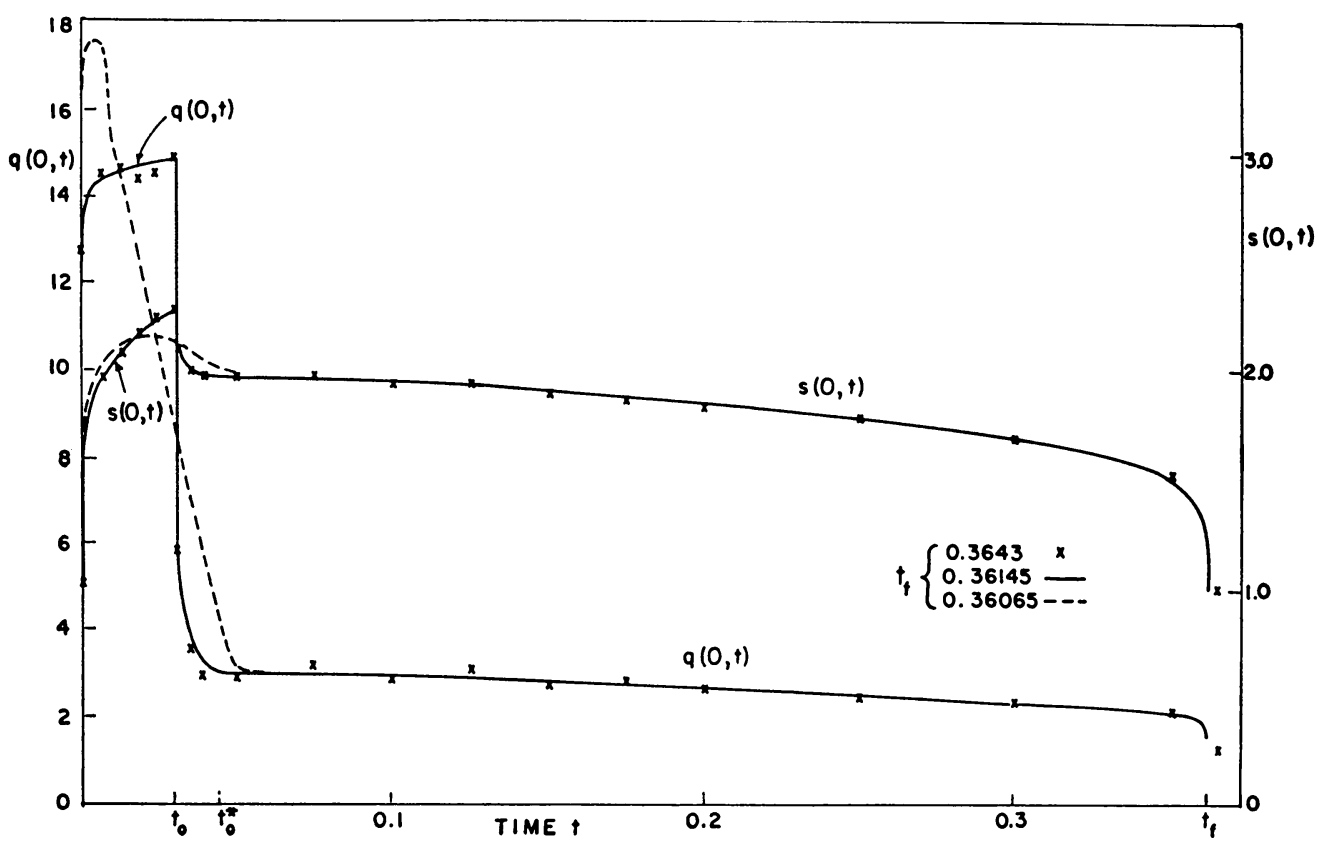

Fig. 3. The Shear and Moment at the Base of the Beam. 


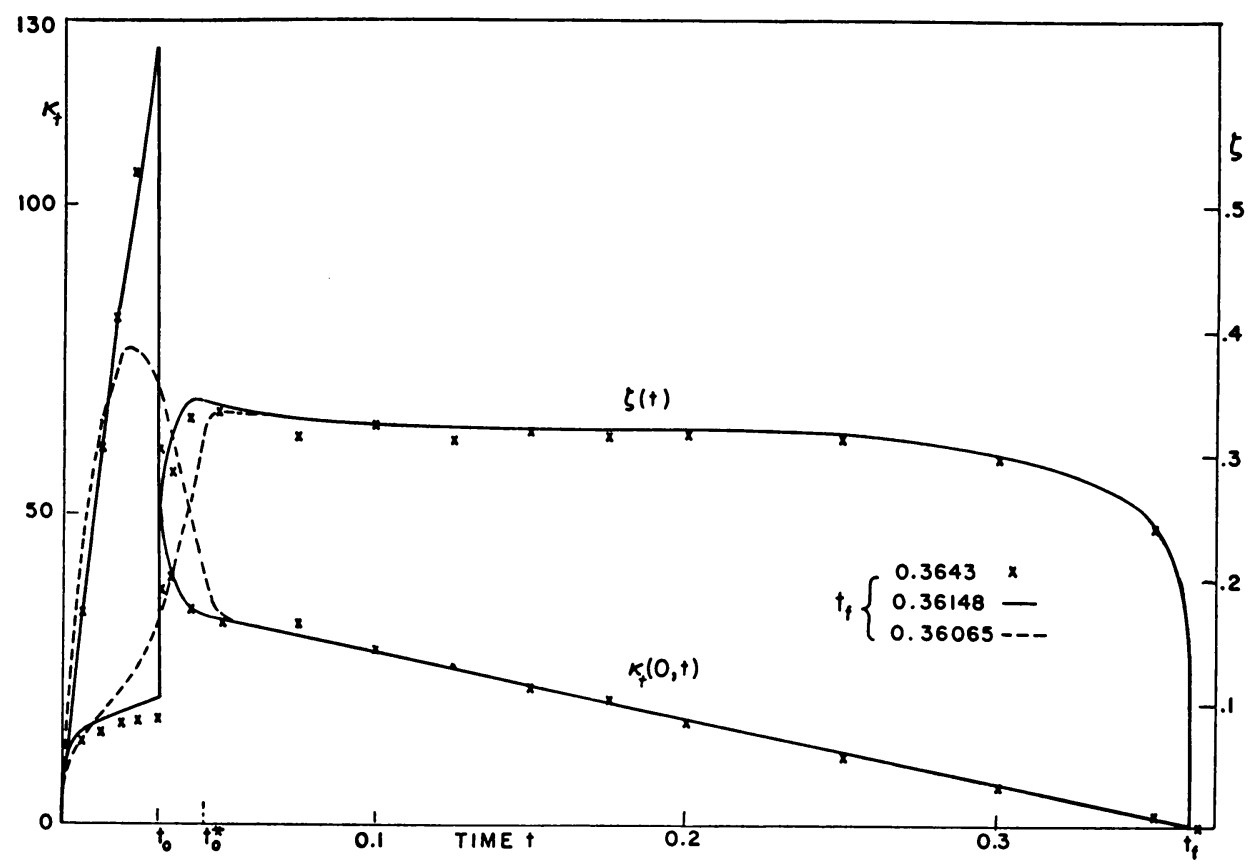

Fig. 4. Rate of Curvature at the Base and the Length of the Plastic Region.

where $c=(k+1)\left[v_{0} f_{t}(0)\left(\frac{1}{2}+k\right)-1\right] /\left(\frac{1}{3}+k\right)$, and $q_{0}=q(0,0)$. In our numerical calculation, (34) is used for the approximate values of $q(0, t)$ and $\kappa_{t}(0, t)$ at $t=\Delta t$ and $2 \Delta t$.

If $f(t)$ is linear between $0 \leq t \leq t_{0}$, abrupt changes in $q(0, t)$ and $\kappa_{t}(0, t)$ occur at $t=t_{0}$ (see Figs. 3 and 4). The approximate values of $q(0, t)$ and $\kappa_{t}(0, t)$ at $t=t_{0}+\Delta t$ also need special treatment. In our calculation, we determine these approximate values by using the simplified equations of motion derived in [11]. The numerical procedure is omitted here (for detail, see [11]). These simplified equations of motion also serve to determine the approximate values of $q(0, t)$ and $\kappa_{t}(0, t)$ used whenever we change the time increment $\Delta t$.

Similarly, a special scheme could be derived to treat the end of the motion $t=t_{\text {s }}$ (see Figs. 1, 3, 4 and 5). However, this is unnecessary as far as the final angle $\theta\left(1, t_{f}\right)$ is concerned. We will show an approximate way to determine the values at $t=t_{f}$.

Since $v\left(x, t_{f}\right)=0, v_{x}\left(0, t_{f}\right)=v_{x x}\left(0, t_{f}\right)=\kappa_{t}\left(0, t_{f}\right)=A\left(0, t_{f}\right)=0$, and $\theta_{t}\left(1, t_{f}\right)=0$ by (7). However, $q\left(0, t_{f}\right) \neq 0$, as we will see later. Equation (9) cannot be satisfied if there is a finite plastic region at $t=t_{f}$. Hence, $\zeta\left(t_{f}\right)=0$. We could have used this method to prove $\zeta(0)=0$.

Suppose we arrive at a step $t_{\lambda}$ such that $\theta_{t}\left(1, t_{\lambda}\right)$ is very close to zero. The magnitude of $\theta_{t}\left(1, t_{\lambda}+\Delta t\right)$ at the next step can be estimated by

$$
\theta_{t}\left(1, t_{\lambda}+\Delta t\right) \cong 2 \theta_{t}\left(1, t_{\lambda}\right)-\theta_{t}\left(1, t_{\lambda}-\Delta t\right)
$$

If the right hand side of the equation is negative, it is clear that $\theta_{t}\left(1, t_{f}\right)=0$ occurs at $t_{\lambda}<t_{f}<t_{\lambda}+\Delta t$. Assuming that $\theta_{t}(1, t)$ is linear near $t=t_{f}$, we can obtain $t_{f}$ approximately by 


$$
t_{f} \cong t_{\lambda}+\Delta t_{f}
$$

where

$$
\Delta t_{f}=\frac{\theta_{t}\left(1, t_{\lambda}\right)}{\theta_{t}\left(1, t_{\lambda}\right)-\theta_{t}\left(1, t_{\lambda}-\Delta t\right)} \Delta t
$$

With (35), we have

$$
\theta\left(1, t_{f}\right) \cong \theta\left(1, t_{\lambda}\right)+\frac{\theta_{t}\left(1, t_{\lambda}\right)}{2} \Delta t_{f} .
$$

The shear $q\left(0, t_{f}\right)$ can be obtained by differentiating (5) with the limit $t \rightarrow t_{f}$, as we did for $q(0,0)$. If this is done, we get

$$
q\left(0, t_{f}\right)=\frac{3(2 k+1)}{2(3 k+1)} \cos \theta\left(1, t_{f}\right)
$$

Hence, $q\left(0, t_{f}\right) \neq 0$.

5. Examples. The numerical procedure described in the preceding sections have been programmed for an IBM-7070 electronic computer and several examples have been calculated. We will described the result of a mild steel beam (No. A1 in Table 1 of [11]) with $p=5, \alpha=38.51, k=0, v_{0}=1.329$ and $t_{0}=0.02948$. This example was used also in [11].

$f(t)$ is regarded as linear in the first phase of the motion $0 \leq t \leq t_{0}$. Ten steps are taken in this phase. Therefore the time increment in the first phase of the motion is $\Delta t_{1}=t_{0} / 10$.

In the second phase of the motion $t_{0} \leq t \leq t_{f}$, approximately thirty steps are taken. Although we do not know the exact value of $t_{f}$ at the beginning, we can estimate it roughly as follows. Suppose the mean value of $s(0, t)$ for $0 \leq t \leq t_{f}$ is 1.8 as found in an example. Then equation (5a) gives

$$
\left(\frac{1}{2}+k\right) v_{0}-1.8 t_{f}=0, \quad \text { or } \quad t_{f}=\left(\frac{1}{2}+k\right) v_{0} / 1.8 .
$$

Therefore, the time increment $\Delta t_{2}$ in the second phase of the motion is $\Delta t_{2}=\left(t_{f}-t_{0}\right) / 30$. Usually, $\Delta t_{2}>\Delta t_{1}$. If it is not, we take $\Delta t_{2}=\Delta t_{1}$.

At $t=t_{0}$, we do not change the time increment from $\Delta t_{1}$ to $\Delta t_{2}$ directly. As shown in Figs. 3 and 4 , abrupt changes occur in $q(0, t)$ and $\kappa_{t}(0, t)$ after $t=t_{0}$. A smaller time increment (much smaller than $\Delta t_{1}$ ) has to be taken in order to obtain accuracy. In our example, we use $\Delta t_{1}$ as a tentative time increment for the first step after $t=t_{0}$ and the approximate values of $q(0, t)$ and $\kappa_{t}(0, t)$ are calculated by the simplified equations of motion in [11]. If $\kappa_{t}\left(0, t_{0}+\Delta t_{1}\right)$ so obtained is smaller than $\kappa_{t}\left(0, t_{0}\right) / 2$, we reduce the time increment by one half. Repeat this process until for a certain time increment, say $\Delta t_{0}$, the approximate value of $\kappa_{t}\left(0, t_{0}+\Delta t_{0}\right) \geq \kappa_{t}\left(0, t_{0}\right) / 2$. $\Delta t_{0}$ is related to $\Delta t_{1}$ by

$$
\Delta t_{0}=\left(\frac{1}{2}\right)^{n} \Delta t_{1}
$$

if we have repeated the process $n$ times. This new time increment $\Delta t_{0}$ is used consecutively for four times after $t=t_{0}$. After this, we double the time increment in each step to obtain the succeeding value, until $\Delta t$ is equal to or just about to exceed $\Delta t_{2}$. Then, $\Delta t_{2}$ is taken as the time increment for the rest of $t$.

$\Delta x$ is taken to be $1 / 100$ throughout the calculation. 


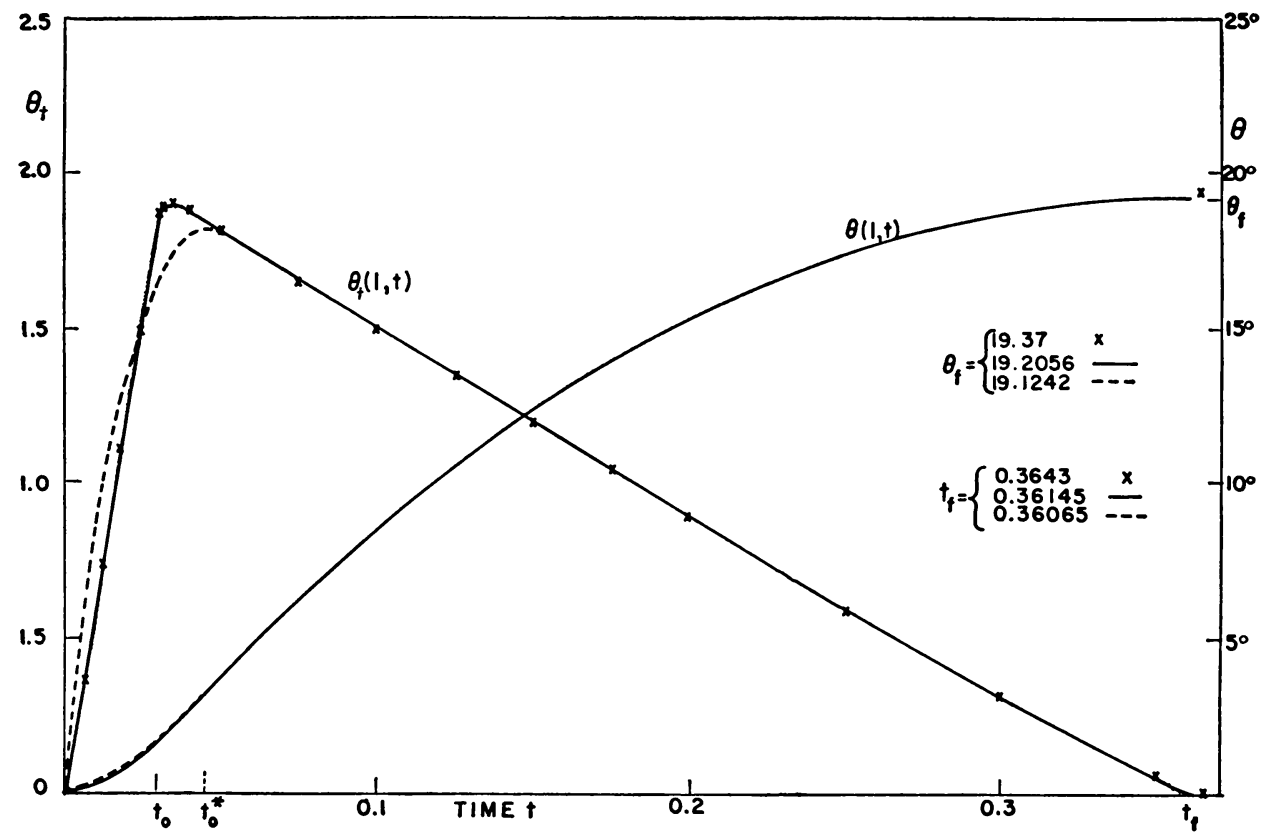

Fig. 5. Change of Slope at the Tip of the Beam.

The final results of the specimen No. A1 are shown in Figs. 3, 4, and 5 by the solid lines. In this example, $\Delta t_{1}=0.002948, \Delta t_{2}=0.01133$ while $\Delta t_{0}=0.0003685$. The result shows that there are 8 steps between $t=t_{0}=0.02948$ and $t=0.04201$ with varying time increments and 28 steps between $t=0.04201$ and $t=t_{f}=0.36145$ with time increment $\Delta t_{2}$. For comparison, the solution obtained by the simplified equations of motion in [11] (shown in Figs. 8, 9 and 10 of [11]) are plotted here with points shown as crosses. It is seen that the agreement between these solutions is very good.

While the results on the boundary $x=0$ are shown in Figs. 3, 4 and 5, those for points inside the plastic region are shown in Figs. 6, 7 and 8, for some particular times. Fig. 7 shows that the moment $s(x, t)$ is nearly linear in $x$ in the plastic region.

It was stated in Section 3 that, at each step, the convergence of the successive approximation in the first stage of calculation is assured if the condition (21) is not violated. To check this, we put $x_{i}=0, t=t_{0}=0.02948$ in $(21)$, since $A\left(x_{i}, t\right)$ is the largest at this point. The left hand side of (21) gives

$$
\frac{5 \times 38.5 \times(0.01)^{4}}{5 ! \times 0.002948}(1.2664)^{4}=0.14 \times 10^{-4}
$$

which is much smaller than one. The rate of convergence is very satisfactory. In the second stage of calculation, the corrections $\delta q$ and $\delta \kappa_{t}$ for $q(0, t)$ and $\kappa_{t}(0, t)$ are calculated. In our example, if the ratio $\delta q / q(0, t)$ or $\delta \kappa_{t} / \kappa_{t}(0, t)$ is larger than $1 / 1000$, the calculation is repeated. It was found that there is no single step which requires more than four repetitions for this accuracy.

The total energy $E_{p}$ absorbed in the plastic deformation is equal to the initial kinetic energy minus the work done by the shear at the base, i.e., 


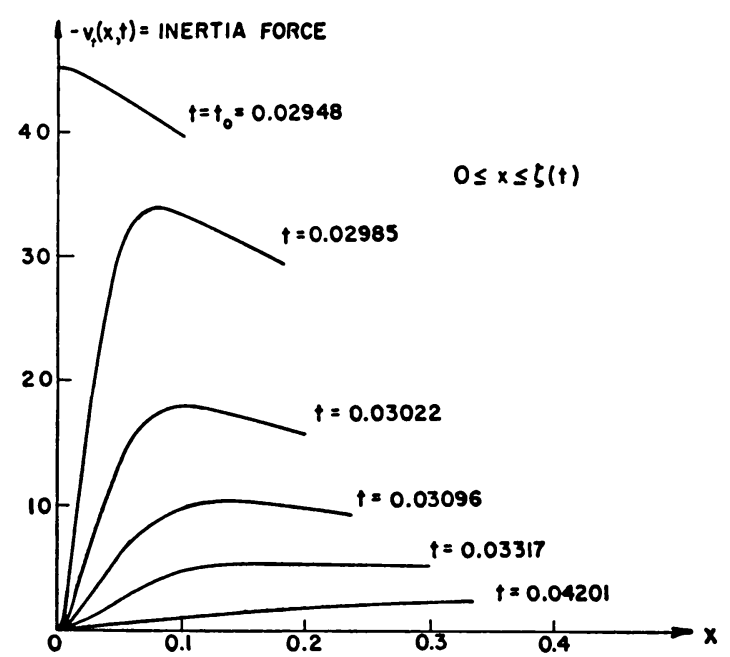

Fig. 6. Inertia Forces in the Plastic Region.

$$
E_{p}=(1+k) v_{0}^{2} / 2-\int_{0}^{t_{0}} q(0, t) v(0, t) d t .
$$

By the mean value theorem, we have

$$
\begin{aligned}
E_{\mathrm{p}} & =(1+k) v_{0}^{2} / 2-q(0, \eta) \int_{0}^{t_{0}} v(0, t) d t \\
& =(1+k) v_{0}^{2} / 2-q(0, \eta) v_{0} \int_{0}^{t_{0}}[1-f(t)] d t
\end{aligned}
$$

where $q(0, \eta)$ is the mean value with $0 \leq \eta \leq t_{0}$.

The assumption that $f(t)$ is linear in $0 \leq t \leq t_{0}$ is quite artificial. It is reasonable to expect that $\theta\left(1, t_{f}\right)$ should be affected very little, if any, by the form of $f(t)$ as long as one keeps the total energy $E_{p}$ fixed. It must be noted that $q(0, \eta)$ does depend on $f(t)$.

In the previous example (specimen No. A1), $f(t)$ was taken to be linear so that

$$
v(0, t)=v_{0}\left[1-t / t_{0}\right] \text {. }
$$

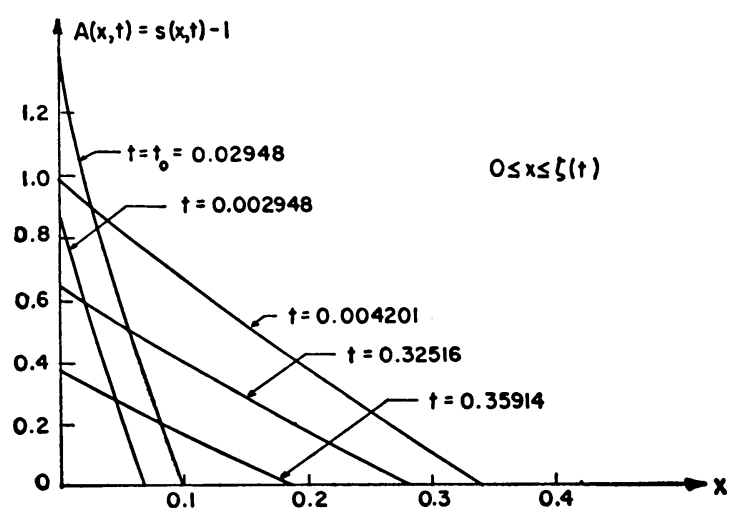

FIg. 7. The Moment in the Plastic Region. 


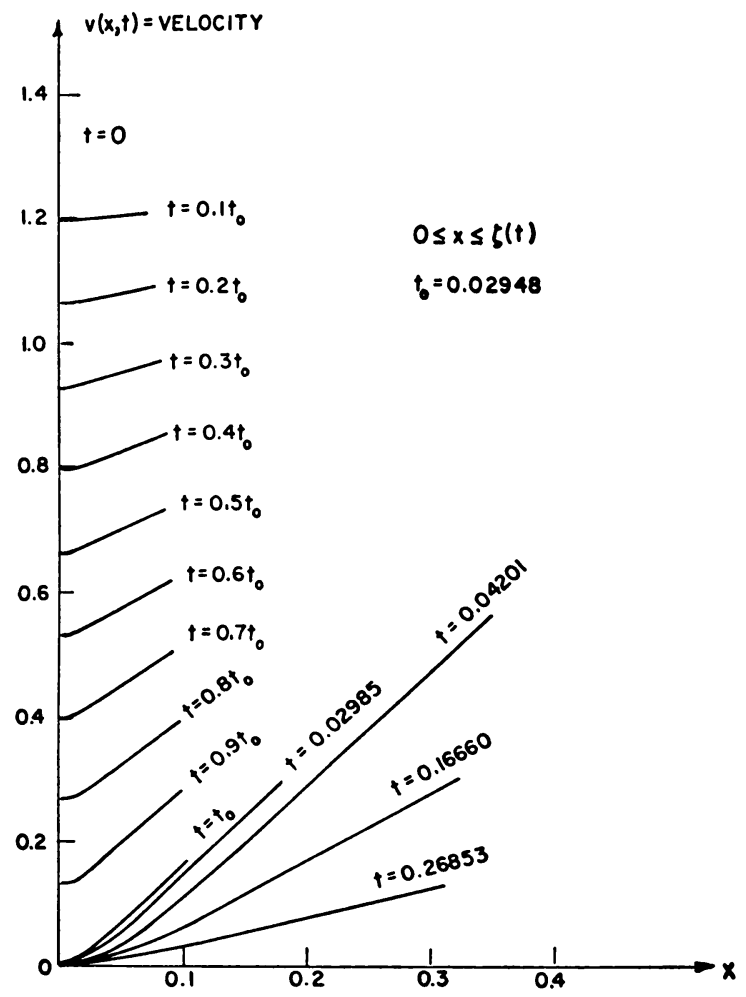

Fig. 8. The Velocity in the Plastic Region.

Let us consider $f(t)$ in a parabolic form. Then the new $v(0, t)$, denoted by $v^{*}(0, t)$, is expressed by

$$
v^{*}(0, t)=v_{0}\left[1-t / t_{0}^{*}\right]^{2}
$$

where $t_{0}^{*}=3 t_{0} / 2$ so that (see Fig. 2)

$$
\int_{0}^{t_{0} *} v^{*}(0, t) d t=\int_{0}^{t_{0}} v(0, t) d t .
$$

With the change of (39), specimen No. A1 is again taken as an example and the results are shown by the dotted lines in Figs. 3, 4 and 5. Although the condition (40) does not assure the same value of $E_{\nu}$ expressed by (38), it was found that the mean values $q(0, \eta)$ differ very little from each other in this example. The results show that $\theta(1, t)$ is practically indistinguishable from the previous one, and the final angle $\theta\left(1, t_{f}\right)$ is very close as we expected.

Three more examples have been calculated with $f(t)$ in the linear form. The final angles $\theta\left(1, t_{f}\right)$ of these three examples are shown in the following table. $\left(\theta_{f}\right)_{I}$ is the angle calculated by the method of [11]. (Eqs. 20, 21 and 23 of [11]), $\theta_{f}$ is the first order approximation of [11] and the last column of the table is the experimental results from [11].

6. Conclusions. This paper presents the solution by an iterative technique of the non-linear fourth order equation of parabolic type, which applies in the region $0 \leq x \leq \zeta(t)$ in which plastic deformation is occurring at time $t$ in a beam with rate- 
TABLE 1. Comparison with the Simplified Theorem [11].

\begin{tabular}{ccccc}
\hline Specimen & $\theta\left(1, t_{f}\right)$ & $\left(\theta_{f}\right)_{I}$ & $\theta_{f}$ & $\left(\theta_{f}\right)_{\text {experiment }}$ \\
\hline Mild Steel A1 & 19.21 & 19.4 & 19.8 & 18.5 degrees \\
Mild Steel B2 & 44.53 & 44.7 & 42.9 & 50.0 \\
Aluminum D3 & 73.64 & & 71.9 & 73.2 \\
Aluminum D13 & 58.63 & & 57.4 & 59.2 \\
\hline
\end{tabular}

dependent yield moment. The results are compared with those obtained by solving the much simpler equations (see [11]) in which inertia forces in the plastic region are neglected. Remarkably close agreement is found between the results of the two solutions. Physically, the inertia forces should be negligible if they are small compared to the shear forces in the plastic region. Then the shear force is nearly constant, and the bending moment varies approximately linearly through the plastic region. The results (Fig. 7) of the present analysis show that the bending moment in the complete solution does indeed vary in a closely linear manner.

The equations of motion dealt with in this analysis and [11] are essentially the equations of conservation of momentum. Neglect of the inertia forces does not imply neglect of the total momentum in the plastic region. In fact, the results show that only a small part of the total momentum is neglected, so that the solutions are practically unaffected when one neglects the inertia forces in the plastic region.

This analysis also shows that the final angle of deformation $\theta\left(1, t_{f}\right)$ does not depend strongly on the form of $f(t)$. The angle $\theta\left(1, t_{f}\right)$ apparently depends mainly on the initial velocity $v_{0}$ and the integral $\int_{0}^{t_{0}} v(0, t) d t$, as indicated by an example.

Acknowledgment. The author wishes to thank Professor P. S. Symonds for his guidance throughout this work. He also wishes to thank the staff members of the Brown University Computing Laboratory, in particular, Mrs. Barbara Friend, for helpful assistance in the execution of the computation presented in this analysis.

\section{References}

1. L. W. Ehrlich, A numerical method of solving a heat flow problem with moving boundry, J. Assoc. Comput. Mach. 5 (1958) 161

2. J. Douglas, Jr. and J. M. Gallie, Jr., On the numerical intergation of a parabolic differential equation subject to a moving boundry condition, Duke Math J., 22 (1955) 557

3. D. Young and L. Ehrlich, On the numerical solution of linear and non-linear parabolic equations on the Ordvac, Interim Technical Report No. 18, Office of Ordnance Research Contract DA-36-034ORD-1486, University of Maryland, February 1956.

4. S. D. Conte and W. C. Royster, A Study of finite difference approximationa to a fourth order parabolic differential equation, Ballistic Research Laboratories, Aberdeen Proving Ground, Maryland, Report No. 959 , Oct. 1955

5. G. G. O'Brien, M. A. Hyman and S. Kaplan, A study of the numerical solution of partial differential equations, J. Math. Phys. 29 (1951) 223

6. F. B. Hildebrand, Methods of Applied Mathematics, Prentice-Hall, Inc. 1954

7. J. Douglas, Jr., On the realtion between stability and convergence in the numerical solution of linear parabolic and hyperbolic differential equations, J. Soc. Indust. Appl. Math. 4 (1956) 20

8. J. Crank and P. Nicolson, A Practical method for the numerical evaluation of solutions of partial differential equations of the heat conduction type, Proc. Camb. Phil. Soc., 43 (1947) 50

9. M. L. Juncosa and D. Young, On the Crank-Nicolson procedure for solving parabolic partial differential equations, Proc. Camb. Phil. Soc. 53 (1957) 448 
10. E. C. DuFort and S. P. Frankel, Stability conditions in the numerical treatment of parabolic differential equations, Math. Tables and Other Aids to Comp. 7 (1953) 135

11. Thomas C. T. Ting and P. S. Symomds, Impact of a cantilever beam with strain rate sensitivity, Proceedings of the Fourth National Congress of Applied Mechanics (June 1962), ASME, New York 1962, Vol. 2, p. 1153 